\title{
Prophylaxis of Hepatitis A with Immune Globulin
}

\author{
Major M G Braithwaite \\ $M B, C h B$, RAMC \\ Specialist in Aviation Medicine \\ Army Air Corps Centre, Middle Wallop
}

SUMMARY: Hepatitis has been outstanding as a military disease. Although it was not recognised as a $\overrightarrow{0}$ commonplace illness in civilian populations until 35 years ago, hepatitis has been a problem in all major: wars during the last two centuries. Much of the knowledge of this disease has been obtained by investigation $\vec{\omega}$ of outbreaks in military populations. The military importance of hepatitis during World War II led to? studies of this disorder which defined it as a distinct clinical entity and delineated both the epidemiology and its mode of transmission.

\section{Introduction}

Pooled Human Normal Immune Globulin (HNIG) was first demonstrated to have an attenuative and prophylactic effect on infectious hepatitis in 1945. It has since been the mainstay of protecting presymptomatic contacts from developing clinical disease. Although administered occasionally to visitors to endemic areas, no definitive policy has been recommended for prophylactic use within the British Arisy

The aim of this paper is to review the use of HNIG in the prophylaxis of hepatitis A and to suggest recommendations for future Army policy. Although HNIG will partially protect against Hepatitis $B$ and possibly against non- $A$, non-B hepatitis, these entities will not be discussed in detail.

A rational approach to HNIG prophylaxis requires an understanding of the clinical virology of hepatitis $A$ and its epidemiology. Once these have been defined, control of the disease and the role of HNIG will be discussed.

\section{Clinical Virology}

Stimulated by research into hepatitis B, most of the virological advance in hepatitis $A$ has occurred in the last decade. A combination of technical innovation in the laboratory, clinical observation under controlled conditions and experiments with primates has, since 1973, brought about great improvements in the knowledge of hepatitis A. It is now known to be caused by an enterovirus which may be isolated from the faeces.

Typical hepatitis $\mathbf{A}$ infection can be defined as an acute viral infection in which jaundice develops about four weeks after exposure and is preceded by several days of malaise during which the virus is excreted in the faeces. Hepatitis A antigen (HAV) is located on the virus and may be demonstrated in the faeces

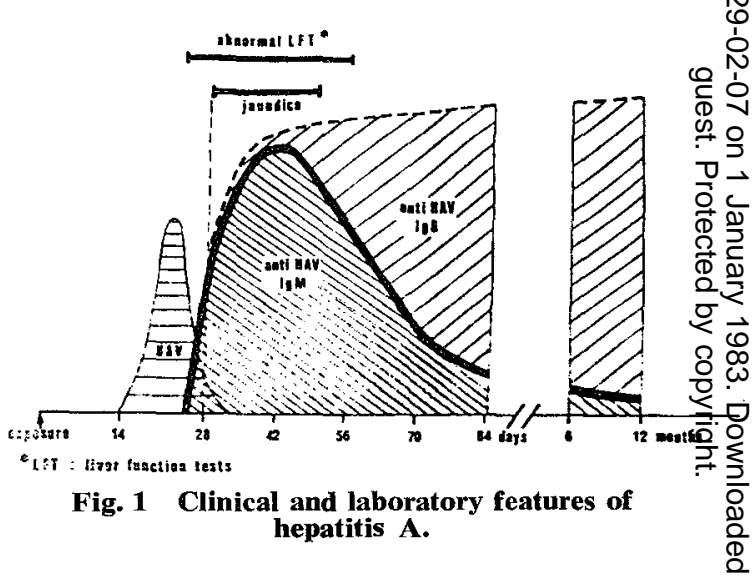

during the incubation period. It disappears with the $\vec{\circ}$ onset of clinical illness and is not found in the 3 blood at any time. The appearance of jaundice is accompanied by abnormal liver function tests and $a ?$ sharp rise in titres of antibodies to HAV of both $\overline{3}$. IgM and IgG classes. (Fig 1). The IgM response is short lived and it usually disappears from the blood within 12 months. IgG antibody persists and confers ${ }_{\widetilde{\infty}}^{-}$ life long immunity to infection. A rising antibody titre of either class or the presence of high levels of IgM defines a primary immune response and is diagnostic of recent infection. Measurement of anti-i HAV of the IgG class can be used in epidemio- $\frac{O}{3}$ logical studies of the prevalence of hepatitis $\mathrm{A}_{\circ}$ infection.

Transmission of the virus is primarily by the faecal $\frac{D}{O}$ oral route. However, past studies have suggested that transmission may rarely occur through water supplies, 0 infected milk and shellfish. The latter are merelyn concentrating vectors and do not act as secondary N hosts. The virus has not been isolated from saliva 
urine, tears, semen or cervical secretions. No chronic carrier state is apparent as is the case in hepatitis B. The illness is generally mild and of short duration. The mortality rate in developed countries is $0.2 \%$ in young adults, death occuring from fulminant hepatic failure in the rare instance.

It is now possible to detect HAV in the faeces from affected patients and to titrate the levels of IgM antibody in the acute phase and IgG antibody which persists in the blood. The assessment of active immunity to hepatitis $A$ is of particular relevance to this discussion and will be considered in detail later.

\section{Epidemiology}

Because of its primary route of transmission by faecal-oral spread, the prevalance of hepatitis $A$ is naturally greater in areas of overcrowding and poor sanitation. Rural areas in the developing countries are greater affected. Within a community infection tends to be introduced by the young children who generally react very mildly to the disease. Infection is propagated by the serial passage of newcomers into an area as the local population rapidly develops immunity. Food-borne and water-borne transmission of hepatitis $A$ is recognised but makes a small contribution to the overall incidence. A classic example of the latter was the epidemic in New Delhi in 1955 when 30,000 cases were reported. Faecal contamination of the water supply was incriminated following severe flooding.

Once tests for anti-HAV had been introduced, laboratories in many countries carried out surveys to assess the prevalence of hepatitis $\mathbf{A}^{1}$. The results provide a useful overall picture but surveys have been small, the groups studied sometimes not representative and the ages of the subjects often not recorded. Several patterns of prevalence emerge from the surveys so far reported. (Table I) The first exists in countries with poor sanitation (not represented in the

Table I

Prevalence of anti-HAV in young adults (20-29 years)

\begin{tabular}{llccc}
\hline Country & Centre & $\begin{array}{c}\text { Number } \\
\text { Exam- } \\
\text { ined }\end{array}$ & $\begin{array}{c}\text { Number } \\
\text { anti- } \\
\text { HAV } \\
\text { Positive }\end{array}$ & $\begin{array}{c}\% \\
\text { Pos }\end{array}$ \\
\hline Sweden & Gothenburg & 208 & 6 & 3 \\
Norway & Oslo & 80 & 4 & 5 \\
Switzerland & Bern & 147 & 18 & 12 \\
UK & London & $220^{*}$ & 28 & 13 \\
Netherlands & Amsterdam & 199 & 62 & 31 \\
W. Germany & Tubingen & 221 & 79 & 36 \\
Belgium & Leuven & 224 & 100 & 45 \\
France & Paris & 107 & 57 & 53 \\
Italy & Milan & 99 & 79 & 80 \\
Greece & Athens & 92 & 77 & 84 \\
Spain & Barcelona & $?$ & - & 90
\end{tabular}

* Voluntary service overseas personnel table), where transmission by the faecal-oral route is unimpeded. In such conditions at least $90 \%$ of the 3 adult population can be expected to be immune to $\stackrel{\varnothing}{\varrho}$ hepatitis A. Although few studies have been carried $O$ out in the tropics it appears that most children are응 infected at an early age. The virus is endemic and $\stackrel{\oplus}{\text {. }}$ susceptible adults, for instance visitors from Europe, $\overrightarrow{\overrightarrow{\mathrm{W}}}$ are at considerable risk of being infected especially if they live amongst the local population.

The second prevalence patiern is that seen in much $\frac{\bar{\sigma}}{\bar{\omega}}$ of Southern Europe. Because of variable social con- $\bar{\phi}$ ditions and hygienic standards, part of the population $\cong$ is infected, mostly subclinically in early childhood. œ Another part is infected mainly with accompanying $\vec{O}$ signs of clinical hepatitis as older children and young $\vec{\overrightarrow{ }}$ adults. This pattern exists in much of Greece, Italy and Spain. In these areas the prevalence of anti-HAV rises steadily to a level of $90 \%$ in middle age.

The third prevalence pattern is one that is probably new to human populations. Studies in developed coun- $\vec{N}$ tries, notably Scandinavia, suggest that the transmis- o sion of hepatitis $A$ is now so inconstant that the disease would disappear were it not for the visits that many make to countries where hepatitis $A$ is endemic: The prevalence of anti-HAV in young adults is now only $10 \%$ in Sweden and not much higher in Lo요 don.

Both notification data and serological studies poime to a change in the epidemiology of hepatitis $A-$ North West Europe and North America in the lagt $\infty_{\infty}$ 20 years. Notifications of infectious jaundice 魚. England and Wales over the 10 years to 1978 a consistent with the fact that hepatitis A no longer circulates freely in this country (Table II). There has been a sharp fall in notifications particularly in the period 1969-1974, and the decline is most pro- $\propto$ nounced in children. The incidence of hepatitis $A$ in $\overrightarrow{0}$ adults has not changed to the same extent, because, 3 while exposure is less common, fewer adults are now immune. Another factor in sustaining the incidence of hepatitis $\mathbf{A}$ in adults is that a relatively large number of infections are now contracted abroad.

Table II

Notifications of infectious jaundice to OPCS

\begin{tabular}{|c|c|c|c|}
\hline & 1969 & 1974 & 1978 \\
\hline $\begin{array}{l}\text { Notifications of } \\
\text { infectious jaundice }\end{array}$ & 23,572 & 7609 & 4663 \\
\hline that are cases $<15$ years & $59.8 \%$ & $41.5 \%$ & $24.5 \%$ \\
\hline
\end{tabular}

The reason for the change in incidence of hepatitis $A$ in the recent past is not entirely plain. It is pro- $N$ bable that the hold of a virus that circulates by the faecal-oral route in a society which generally enjoys 0 basic sanitary services and a safe water supply is $\omega$ 
always tenuous and that a small improvement in habits of personal hygiene, particularly among children can lead to a disproportionate change in the prevalence of hepatitis A. Improvements in the toilet facilities in primary schools and the use of HNIG to prevent secondary cases may account for the change. However, the elimination of some unrecognised route of transmission in recent times may be another explanation.

\section{Control of Hepatitis A}

As a world problem, improvement in hygiene standards must be considered primarily in the control of hepatitis A. Provision and maintenance of sanitation services in developing countries is paramount. For the individual traveller, personal toilet and eating habits should be impeccable. When travel off the beaten track is contemplated, communal living with native populations should be avoided or controlled so as to maintain a civilised standard of hygiene. Notwithstanding these general measures, immune globulin prophylaxis is available in most developed countries.

Immune globulin was first used in connection with infectious hepatitis in 1945. Its efficacy in preventing secondary cases developing during an epidemic in an American children's home was well proven. Following this demonstration it proved valuable in reducing the prevalence of hepatitis in American soldiers in the Mediterranean at the end of World War II. Many early studies repeatedly proved its effectiveness in arresting the spread of the disease within small communities once an index case had been identified. Indeed post-exposure prophylaxis has been the main use of HNIG over the past 37 years and there can be little controversy about this sort of therapy. The ability to detect immunity in the individual has rationalised its use in this way.

However, the indications for using HNIG to protect the individual without an epidemic, i.e. preexposure prophylaxis, is less clear. Encouraging results were obtained from a trial with missionaries and voluntary overseas workers in the late 1960 ' $\mathrm{s}^{2}$. Observed incidence rates in unprotected groups were similar to that of the local population. When HNIG was given, the incidence was greatly reduced over the ensuing six months. At the same time as this study, results from a survey in a mental institution in the USA where hepatitis was common, suggested that the protection was afforded by the development of active immunity from subclinical infection under immune globulin cover. However, this was not evident from serological studies in the endemic areas ${ }^{3}$. Repeated administration of HNIG was necessary to maintain a reduced rate of infection. The failure of this passive-active immunity in these circumstances was probably due to the relatively low attack rate compared to that in the outbreaks where the phenomenon was observed (less $\mathbb{\Phi}$ than $5 \%$ compared to $30 \%$ ).

Following the development of techniques for음 measuring anti-HAV it was possible to assess accurately the protective capacity of HNIG. A Swedish $\Rightarrow$ trial in 1976 examined anti-HAV levels in soldiers $\stackrel{0}{\rightarrow}$ before and after a tour in the endemic areas of 0 Sinai and Cyprus ${ }^{4}$. It concluded that a prophylaxis $\frac{\overline{ }}{\bar{N}}$ scheme of administration of HNIG to non-immune $\frac{\infty}{\sigma}$ deployed personnel was highly effective (one case $\varrho$ of hepatitis $\mathrm{A}$ was seen in 615 innoculated troops). Whilst the results appear excellent, it should be $\vec{A}$ noted that the tour was for a six month period only.

Clearly HNIG is of use in protecting the indivi- $\vec{\omega}$ dual and small populations temporarily deployed to an endemic area. Passive immunity must be augmented every five or six months depending on the potency of the immune globulin. Repeated inocu- $\frac{1}{N}$ lation may be simple for the small group but there $\tilde{C}$ would be considerable logistic difficulties in protecting a large unit for any length of time. Whilet we await the development of a vaccine to stims late active immunity, there is difficulty in deter mining a policy regarding who should receive $\mathrm{HNI} \cdot \overrightarrow{\mathrm{C}}$ and whether screening for immunity prior inoculation is warranted.

\section{A Prophylactic Policy}

There is little sign that the decline of hepatitis as an indigenous infection in parts of North Weg Europe is being matched by changes in endemicit in the developing countries. ${ }^{5}$. Any traveller who visits Africa, Asia or Central or South America is at considerable risk of exposure to hepatitis $A^{6}$. The $\mathbb{Q}$ risk to the holidaymaker who goes to the shores of $\overrightarrow{\overrightarrow{0}}$ the Mediterranean is much smaller; but as several $\frac{0}{3}$ millions of Britons visit there each year, it is not surprising that that is where many imported hepatitis A infections are acquired.

Swedish researchers have recommended that all travellers be given prophylactic HNIG now that susceptibility to hepatitis $A$ is general. They estimate that half of the million Swedes going abroad each year are given HNIG, and observe that almost all cases of hepatitis $A$ arise in those travellers not given prophylaxis. It would at present be impracticable to implement this policy in Britain, and it is open to doubt whether the risk from a fortnight's visit to the Costa Brava warrants the discomfort of the present large intramuscular injection.

The American Immunisation Practices Advisory $N$ Committee recommends that all travellers to high risk areas outside ordinary tourist routes should $\tilde{D}$ receive a single $\mathrm{HNIG}$ dose of $3 \mathrm{mg} / \mathrm{Kg}$ for journeys $\mathrm{C}$ 
up to three months ${ }^{7}$. For more prolonged travel $10 \mathrm{mg} / \mathrm{Kg}$ should be given every five months. Their military medical authorities also recommend that $10 \mathrm{mg} / \mathrm{Kg}$ be given to their Rapid Deployment Force immediately prior to embarkation ${ }^{8}$. No mention is made in either case of testing for immunity prior to inoculation.

As far as the British Army is concerned, we are unlikely to despatch a deployment force of substantial size to an endemic area without integral medical support although there are frequent major unit exercises to high risk areas such as Sardinia and Turkey. Notification data of hepatitis $A$ in troops stationed abroad is confusing. This is due both to a recent change in the International Classification of the hepatidides and the failure to notify. (Some estimate that only $20 \%$ of cases are notified). Whatever the true incidence, most established British overseas postings are well supported by medical facilities so neither is the care of an infected soldier a problem nor is his absence from duty for a limited period likely to interfere with the unit's efficiency. Given this situation, the pre-exposure prophylaxis of major units on well supported tours abroad is not justified.

Our main cause for concern is the Army training expedition and personnel and their dependants on loan service in endemic areas. Medical facilities for the former group rarely exist throughout the course of the expedition and a medical casuality of this nature might seriously embarrass the expedition's objective. The problem of loan service personnel came to light recently following a visit to assess medical facilities of The British Advisory Team ${ }^{9}$. Clinical facilities were by no means comprehensive, and personnel and their dependants did not relish the prospect of spending any length of time in local civilian hospitals. Of course hepatitis $A$ is rarely severe enough to warrant hospital admission but the absence of a home-visiting medical officer means that complications may go unnoticed. Compounding this situation is the natural anxiety of mothers for their young children in a tropical climate. Again notification data of the incidence of hepatitis $\mathbf{A}$ in these groups is unreliable but the number of cases appears proportionately greater than those reported from British bases.

Less than a thousand personnel including dependants are involved in these two special situations ${ }^{10}$. At present administration of HNIG is haphazard. Most commonly the request comes from the individual concerned to his Medical Officer who may or may not prescribe depending on his opinion and the availability of HNIG. A policy to cover all concerned is necessary to determine the need for drug supply and investigative facilities. Before considering a policy it is clear that a more detailed record is needed of the incidence and localities where service personnel contract hepatitis.

The need for pre-exposure prophylaxis has been outlined above. It must therefore be decided whether screening for immunity prior to inoculation is justified. Many of the personnel for whom HNIG prophylaxis is indicated tend to be naturally itinerant. They may already have been exposed to hepatitis $\mathrm{A}$ and developed immunity through clinical or subclinical infection. Therefore the prevalence of anti-HAV within the Army should be determined. A cross-sectional study would help to identify the need for pre-inoculation screening. In the meantime it would appear reasonable to screen inveterate travellers only and to offer prophylaxis to children and non-immune adults.

\section{Cost}

This paper has not attempted to analyse the cost of the proposed policy. However, following the administration schedule recommended by The Central Public Health Authority (CPHA) the financial aspects appear as follows:

(a) HNIG at $£ 5$ per gramme should be given:

1) to children as a $0.25 \mathrm{~g}$ dose.

2) to adults as a $0.5 \mathrm{~g}$ dose. and repeated six-monthly.

(b) This represents a cost for prophylaxis of between $£ 5$ and $£ 10$ for a two year tour. (The costs of transport and storage have not beeno considered).

(c) Anti-HAV screening is currently performed by CPHL. It is unlikely that they will be able to continue this service and it is believed that RAM College will inherit the commitment. Technical equipment is available but test kits will need to be purchased. A cost of $£ 2.10$ per test is likely.

\section{Conclusions}

Hepatitis A is an important disease amongst military populations. Since 1945 Human Normal Immune Globulin (HNIG) has been used effectively in controlling outbreaks of this infection once an index case has been identified. Its efficacy when given prior to exposure is less clear and this is discussed.

Because of its primary route of transmission by faecal-oral spread, an improvement in hygiene standards is of paramount importance in the control of the disease in developing countries. Studies of the prevalence of hepatitis $A$ antibody have shown that immunity is rare in North West Europe. In Asia about $90 \%$ of the population are immune and a similar proportion is reached by middle age in the Mediterranean countries. Infection in popu- 
lations of developed countries are largely as a result of travel to endemic regions. If infection were better controlled the disease may disappear from these areas. This cannot be achieved until active immunisation is available. In the meantime more effective use of HNIG will help.

Although active immunity may develop via subclinical infection under HNIG cover during outbreaks in developed countries, this is not apparent in long-term visitors to endemic areas. Pre-exposure prophylaxis has been effective in reducing the incidence of clinical illness in both small and large emigrant populations for a limited period. No long-term protection is afforded unless inoculation is repeated at five to six monthly intervals. Both Swedish and United States civilian ${ }^{11}$ and military authorities recommend HNIG prophylaxis for travellers to endemic areas.

British major units stationed overseas have good medical support and would cope with the 'normal' incidence of hepatitis $A$. Therefore routine prophylaxis of major units is not recommended. However, the Army training expedition and loan service personnel have limited medical facilities and medical casualties of this nature would be an embarrassment. Although further study is needed of the military incidence of hepatitis $A$ and prevalence of immunity and the offer of prophylactic HNIG to children and non-immune adults is recommended.

\section{Recommendations}

It is recommended that:

(a) Detailed information of the incidence and localities where service personnel contract hepatitis $A$ is obtained.

(b) The prevalence of immunity is assessed within the Army.

(c) Regular travellers to endemic areas be screened for immunity.

(d) Non-immune expedition and loan service personnel and their dependants be offered immune globulin prophylaxis prior to posting and at six-monthly intervals.

\section{Acknowledgements}

I am most grateful to the staff of The Royal Army Medical College library for their inexhaustibled assistance in locating references.

\section{REFERENCES}

1 ZuCKerman, A et al. Recent Advances in Clinica Virology. 1981.

2 Mann, J M AND Ahtoe, J L. Prophylaxis for Hepatitis A. Am Family Phys 1982; 25: 129-132. 으

3 Pollock, $T$ M AND REID, D. Immunoglobulin the Prevention of Infectious Hepatitis in persogs working Overseas. Lancet Feb 8, 1969; 281-283?음

4 WEILAND, $O$ et al. Immunoglobulin Prophyla쪼against Hepatitis A among. Swedish UN soldiers 9rf an Endemic Region. Infection 7. 1979; 5: 223-285;

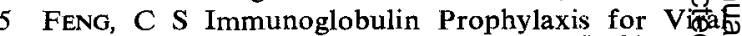
Hepatitis, J Family Pract 1982; 14: 677-681.

6 PHLS. Assessment of British Gammaglobulin preventing Infectious Hepatitis. Br Med J 1982:020 451-454.

7 United States Immunization Practices Advis श्: Committee. Immune Globulins for Protectis against Viral Hepatitis. Ann of Internal Med 19\$2
96: 193-197.

8 Walter REed ARMY INSTITUTE OF RESEARCID๊ Washington D.C., Proceedings of The Workshop of Infectious Disease Threats to The Rapid Deploy ment Force: Preventive Strategies. 1981.

9 Wing Commander Russell, W B. Visit to British Advisory Team Nigeria. RAFSC/452005/4 Med 1982.

10 Quarterly Return of Army Service Personnel om Loan Service to other Countries.

11 Morbidity and Mortality Weekly Report. Vol. 30 No. 34: 1981 . 